\title{
The Kinetics of Titanium Monosilicide Growth Studied by Three-wavelength Ellipsometry
}

\author{
J. M. M. de NIJS* and A. van SILFHOUT \\ Department Solid State Physics, University of Twente, P.O. Box 217, NL-7500 AE Enschede (The Netherlands)
}

(Received June 2, 1989)

\begin{abstract}
Thin titanium layers (approximately $10 \mathrm{~nm}$ ) have been grown on top of a clean Si(111) substrate. Heating these layers initiates a solid state reaction, yielding an amorphous monosilicide phase at about $350{ }^{\circ} \mathrm{C}$. The kinetics of the solid state reaction has been followed using three-wavelength ellipsometry (340, 450 and $550 \mathrm{~nm})$. A very coarse two-layer model has been applied in the analyses of the measured data: a top layer of pure titanium is consumed by a second layer of TiSi. The dielectric constants of titanium and TiSi are known and the layer thicknesses $d_{1}$ and $d_{2}$ have been fitted to the six ellipsometrical angles measured. These analyses reveal a diffusionlimited growth mechanism exhibiting two growth rates: a rapid initial rate followed by a slower final rate. The diffusion coefficient $D$ of the rapid process and its activation energy $E_{a}$ could be obtained: $D=2 \times 10^{-1.5} \mathrm{~cm}^{2} \mathrm{~s}^{-1}$ at $T \simeq 370^{\circ} \mathrm{C}$ and $E_{a}=0.62$ $\mathrm{eV}$. The two growth rates have been attributed to silicon diffusion along the grains and diffusion into the grains.
\end{abstract}

\section{Introduction}

This paper has been written in succession to ref. 1 , in which we have described the phase formation of the $\mathrm{Ti}-\mathrm{c}-\mathrm{Si}$ solid state reaction. Upon heating a thin titanium layer (approximately 10 $\mathrm{nm})$ grown on top of a clean $\mathrm{Si}(111)$ substrate, we observed the growth of a metastable amorphous monosilicide (at about $350{ }^{\circ} \mathrm{C}$ ). In this paper we will discuss the growth kinetics of this monosilicide phase.

\footnotetext{
*Present address: Department of Physics, Eindhoven University of Technology, P.O. Box 513, NL-5600 MB Eindhoven, The Netherlands.
}

The low temperature solid state reaction has already been studied by Tromp et al. [2], Chambers et al. [3], Holloway and Sinclair [4] and Raaijmakers [5]. All these investigations agree on the formation of a monosilicide at low temperature (about $400{ }^{\circ} \mathrm{C}$ ); however, there is a large scatter among the diffusion coefficients reported. Ellipsometry offers the advantages of depth sensitivity (approximately $30 \mathrm{~nm}$ ) [6,7], an accurate and quantitative measurement and the availability of mathematical models that can be utilized in the analysis of the optical data. Ellipsometry is therefore a most appropriate technique in the study of the growth kinetics of a monosilicide layer buried by an unreacted titanium layer.

In the present study we have followed the intermixing process by three-wavelength ellipsometry $(\lambda=340,450$ and $550 \mathrm{~nm})$. Data points comprising the $\Delta$ and $\psi$ values at each wavelength were measured every $30 \mathrm{~s}$. The optical time-resolved measurements of the monosilicide formation could be fitted to a simple planar layer model; a top layer of pure titanium above a monosilicide layer. In this way we could calculate the thickness of the monosilicide layer as a function of time. Because of the immobility of titanium, silicon is shown to be the dominant moving species $[8,9]$, an amorphous silicide being obtained at low temperatures.

From fundamental considerations one can distinguish two growth modes: interface-limited and diffusion-limited growth $[10,11]$. The analyses of our time-resolved data agree with a diffusionlimited process for the monosilicide formation. However, two growth rates are observed: a rapid initial rate followed by a slower final rate The rapid initial growth rate could be an indication of enhanced silicon diffusion through atomically disordered regions. Such a diffusion mechanism has been suggested previously $[2,3,12,13]$. 


\section{Optical analysis of the monosilicide growth}

The growth process is followed ellipsometrically at three wavelengths: 340,450 and $550 \mathrm{~nm}$. The experimental details can be found in ref. 1 . One of the options we had was to measure $\Delta(t)$ and $\psi(t)$ at these wavelengths at a fixed temperature increment and at equal time intervals of $30 \mathrm{~s}$. Figure 1 displays the measurement data, drawn in the $\Delta-\psi$ plane, corresponding to monosilicide growth. Prior to the experiment the sample was annealed for $2 \mathrm{~min}$ at $T \simeq 200^{\circ} \mathrm{C}$, allowing the titanium layer to relax and producing a stable titanium microstructure or constant (approximately zero) voids fraction $[6,7]$. The measurement commenced at a temperature of about $300{ }^{\circ} \mathrm{C}$, which is increased to about $370{ }^{\circ} \mathrm{C}$ after $40 \mathrm{~min}$. The optical data thus comprises six independent quantities, the $\Delta$ and $\psi$ values for each of the three wavelengths. For the analysis of the first diffusion process we have assumed that a planar monosilicide layer grows which then consumes the titanium layer above. Such an intermixing process is easily modelled by a two-layer model, presuming that the dielectric constants are known. The dielectric function of titanium has been determined previously [14] and the dielectric constants on the monosilicide and $\mathrm{c}-\mathrm{Si}$ have been obtained from ref. 1. We can thus fit this two-layer model to the optical data.

The dielectric functions have been measured at room temperature whereas the silicide growth occurs at higher temperatures. This effect is observed in the measurements: $\psi$ is shifted rever-

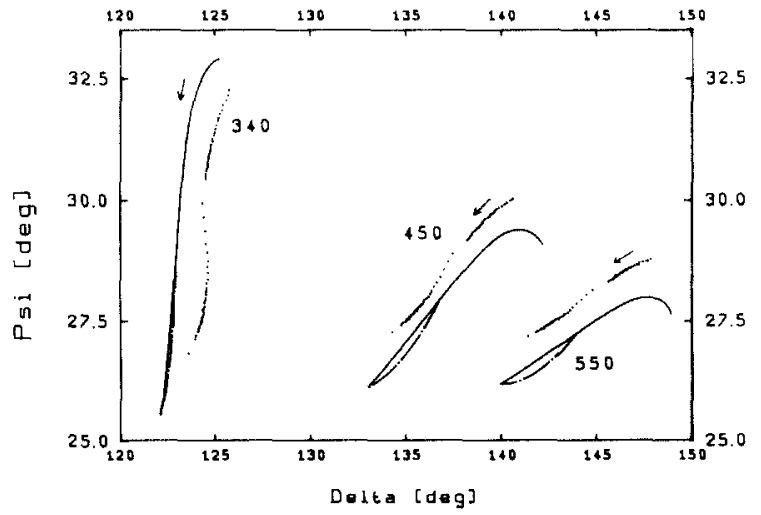

Fig. 1. Trajectories in the $\Delta-\psi$ plane of the Ti-TiSi transformation. The dotted lines denote the experimental data. The solid lines denote the planar growth of a TiSi layer consuming the titanium layer above. The chain lines denote the trajectories of an initially linearly grown TiSi layer, but the final $5 \mathrm{~nm}$ of titanium are converted to $\mathrm{TiSi}$ by a process of mixing. See text and Fig. 4. sibly to an angle of $1^{\circ}$ owing to the temperature increment. Because of this perturbation of the dielectric constants, we have used the very coarse model of two pure layers which do not contain voids, reflecting the microstructure of the layers $[6,7,15]$. One is forced to do so in order to limit the freedom of any solution, thus avoiding errors due to too large an ability to fit the model to the measured data. Hence every time point consisting of six independent quantities is fitted to a twolayer model, yielding two optimized layer thicknesses $d_{1}$ and $d_{2}$. For this purpose we have applied a linear regression analysis (LRA) programme based upon the Levenborg-Marquardt minimization routine [16]. A similar example of such an analysis can be found in ref. 14 .

The analyses of the individual data points comprising $\Delta$ and $\psi$ values at three wavelengths are depicted in Fig. 2 . The result $d_{1}$, the thickness of the titanium layer, is shown as a function of the TiSi thickness $d_{2}$. For the five samples we see that the titanium layer is consumed at the same rate for the initially thinner layers (approximately 8 $\mathrm{nm}$ of titanium) and for the thick ones (approximately $15 \mathrm{~nm}$ of titanium).

From the thickness relation and the initial titanium layer thickness we have calculated the $\Delta-\psi$ curves for the sample depicted in Fig. 1. The calculated curves are denoted by the solid lines. Clearly shown is the large systematic discrepancy between these optimized trajectories and the actual measured data points. The difference has to be ascribed mainly to the temperature effect: the dielectric constants used are those obtained at room temperature whereas the silicide growth occurs at higher temperatures. Errors will be introduced by this shift; however, since the shifts

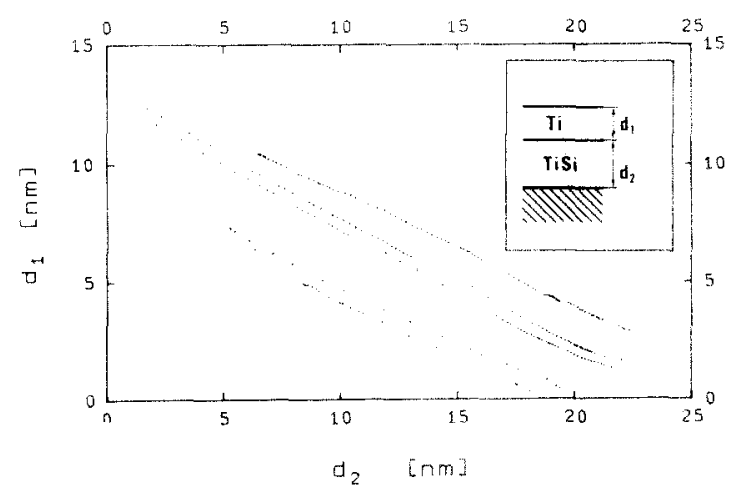

Fig. 2. Thickness of the non-consumed layer $\left(d_{1}\right)$ as a function of the TiSi layer thickness $\left(d_{2}\right)$ as calculated from the data of five experiments. 
are systematic, the errors in the optimized parameters $d_{1}$ and $d_{2}$ will also have a systematic character. It is obvious that optimizing this fraction will yield erroneous results if the layers are allowed to contain a variable voids fraction.

\section{Discussion}

From a simple consideration of the mass transport across an interface and through a layer we postulate that there are two growth modes: an interface-limited mode $\left(d_{2} \propto t\right)$ and diffusioncontrolled mode $\left(d_{2} \propto t^{1 / 2}\right)[10,11]$. In Fig. 3 we have plotted $d_{2}^{2}$ as a function of time for two samples and two temperatures. At $T=300{ }^{\circ} \mathrm{C}$ we see that $d_{2}{ }^{2}$ is proportional to $t$ for both samples. In so far as we could study the growth process (there was already a thin 3-5 nm intermixed region [1]), we did not observe any trace of an interface-limited process.

The growth rate is strongly increased on raising the temperature to 350 or $370{ }^{\circ} \mathrm{C}$. Initially at these temperatures a diffusion-limited growth is followed $\left(d \propto t^{1 / 2}\right)$; however, in both cases we find that the growth rate diminishes although the diffusion process has not yet terminated. Apparently there is a second, slow diffusion process which continues once the fast diffusion process has terminated. We will discuss this slow diffusion process later on and proceed now with the fast process.

The diffusion coefficients at $300{ }^{\circ} \mathrm{C}$ and $370^{\circ} \mathrm{C}$ are $5 \times 10^{-16} \mathrm{~cm}^{2} \mathrm{~s}^{-1}$ and $2 \times 10^{-15} \mathrm{~cm}^{2}$ $s^{-1}$ respectively. This is in good agreement with the result obtained by Chambers et al. [3], who found a value of approximately $7.5 \times 10^{-16} \mathrm{~cm}^{2}$ $\mathrm{s}^{-1}$ at $T=340{ }^{\circ} \mathrm{C}$ for a titanium layer grown from an evaporation source. Raaijmakers [5] has

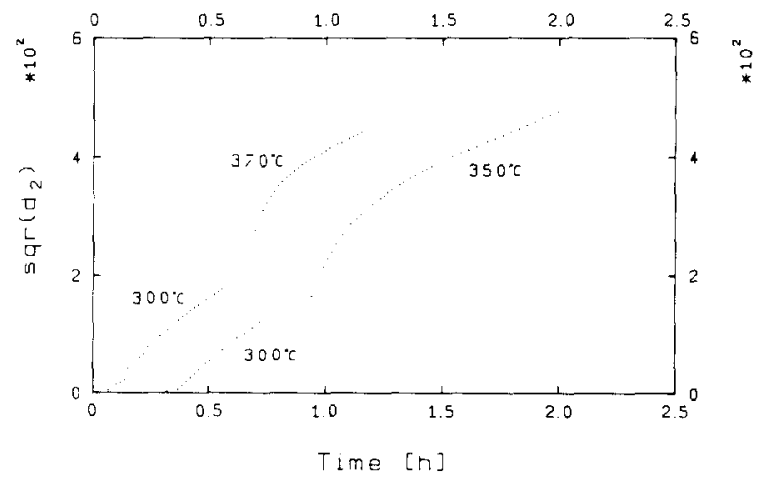

Fig. 3. Squared thickness of the TiSi layer as a function of time for two temperatures. Two experiments are depicted studied the growth kinetics of the a-Si/sputtered titanium system; he found a diffusion coefficient of about $1.0 \times 10^{-16} \mathrm{~cm}^{2} \mathrm{~s}^{-1}$ at $T \simeq 400^{\circ} \mathrm{C}$. Hence the intermixing process in the case of an evaporated titanium layer evolves roughly an order of magnitude faster than the interdiffusion of a-Si and sputtered titanium. Such a discrepancy is too large to be attributed to experimental errors and therefore it can be concluded that the reaction kinetics depends on the nature of the system. In particular, the deposition technique for the titanium layer is expected to affect the titanium film structure strongly, which in turn might affect the diffusion coefficient.

From the two diffusion coefficients we can easily calculate the activation energy for the monosilicide formation. A value of $0.62 \pm 0.1 \mathrm{eV}$ is found, which is somewhat smaller than the value reported by Chambers et al. [3], $E_{\mathrm{a}}=1.0 \pm 0.25 \mathrm{eV}$. Other values have thus far not been reported in the literature, but for $\mathrm{TiSi}_{2}$ (C49) formation an activation energy of about 1.8 $\mathrm{eV}$ is found $[5,17]$. This higher activation energy for the $\mathrm{TiSi}_{2}$ growth explains why the growth of a monosilicide is not observed at high temperatures.

As we have stated, there are at least two diffusion-controlled growth mechanisms: a fast initial one succeeded by a slower second one. Further, we have seen that the diffusion rate is an order of magnitude higher than that for sputtered titanium layers. A possible explanation for both phenomena might be given by the microstructure of the titanium layer.

With regard to high melting point metals, molecular dynamics $[18,19]$ shows that the adatom mobility is low for low substrate temperatures, allowing adsorbed atoms to move no further than to a neighbouring lattice site. Owing to this restricted lateral diffusion, a polycrystalline layer containing numerous lattice defects or even micropores or voids can be expected. A higher substrate temperature and a larger kinetic energy of the arriving atoms reduce the number of defects or improve the structure of the layer. This effect has recently been clearly shown by Yamada and Yoshida [20], who have studied evaporated titanium films grown at various substrate temperatures by means of Röntgen diffraction. Therefore it can be expected that the deposited titanium layer is composed of extremely small grains or clusters a few nanometers in size, separated by regions of disordered atoms. Heating 
such a layer should improve the crystallinity or ordering of the atoms, which in fact is exactly what is observed $[6,7]$. Moreover, sputtered titanium layers are expected to have a better structure (larger grains) than that of evaporated films, since in the latter case the atoms arrive with little thermal energy, whereas sputtered atoms do have a kinetic energy of the order of $1 \mathrm{eV}$.

It is frequently suggested that grain boundaries provide short circuits for silicon diffusion through a metal layer $[2,3,10]$. Hard proof, such as for some metal-metal systems $[9,21]$, is not available. However, if we assume that silicon diffusion is enhanced with diminishing film structure, it offers an explanation for the two growth rates and for the anomalous low diffusion coefficient found by Raaijmakers [5]. The presently studied layers have an extremely fine polycrystalline or cluster structure. The initial silicon diffusion will prefer the disordered regions and a monosilicide will grow predominantly in the disordered regions. This preferential growth will terminate owing to lack of disordered titanium, whereas the retarded in-diffusion into the ordered regions (grains or clusters) continues. This second process can be clearly observed in the absence of the fast diffusion.

In our analysis of the experimental data we have applied a simple planar layer model. The discrepancy between this model and the proposed diffusion mechanism will be obvious, and therefore it is required to determine whether or not we can distinguish optically between the simple planar layer model and a more advanced model in better agreement with the proposed diffusion mechanism.

Once the silicon diffusion along the grain boundaries has reached the titanium surface and all the disordered titanium has been consumed, this growth mode terminates whereas the diffusion of silicon into the titanium grains or clusters continues. Since the grains near the interface are longer exposed to the high silicon concentration, they are converted to TiSi to a greater degree than are the grains near the surface. Therefore this final process of silicon diffusion into the grains is mainly observed near the surface of the sample, and if so, it looks like a mixing of titanium and TiSi above an already converted TiSi layer. We illustrated this process in Fig. 4. Such a process seems in better agreement with the proposed diffusion mechanism and can be modelled optically. We have assumed that initially a normal layer growth commences, but that the final $5 \mathrm{~nm}$ of titanium are not consumed in a planar manner from below but mix homogeneously with $\mathrm{TiSi}$; the Ti:TiSi ratio diminishes from unity to zero. Meanwhile, the volume expansion, or thickness, of this top region increases linearly with the TiSi amount. For these calculations we have applied the Bruggeman effective medium approximation. The trajectories in the $\Delta-\psi$ plane thus obtained for the final growth process are displayed by the chain lines in Fig. 1. From these results we see that these trajectories are only slightly affected and that it is difficult to distinguish between an ordinary planar layer growth and the presently discussed terminal stage.

\section{Conclusions}

In this paper we have studied the growth kinetics of low temperature monosilicide growth. Silicon transport is diffusion limited and a growth proportional to $t^{1 / 2}$ was observed. The diffusion coefficient at $300^{\circ} \mathrm{C}$ is $5.0 \times 10^{-16} \mathrm{~cm}^{2} \mathrm{~s}^{-1}$ and the activation energy $0.62 \mathrm{eV}$. This diffusion coefficient is approximately an order of magnitude larger than that reported by Raaijmakers [5]. This large discrepancy may indicate that the silicon transport is strongly affected by the titanium microstructure.
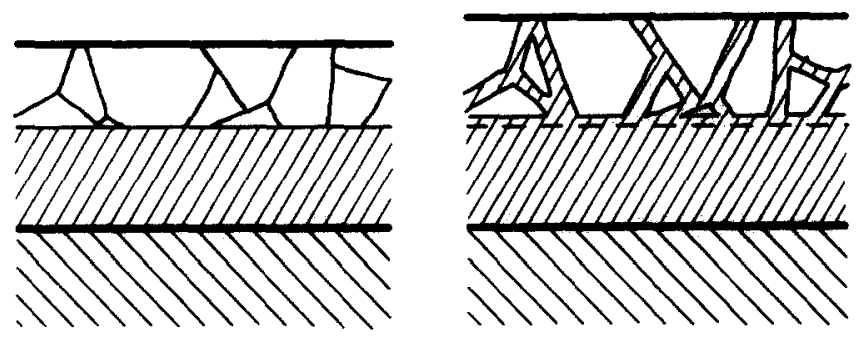

Fig. 4. Schematic illustration of Ti-TiSi mixing in the top layer.

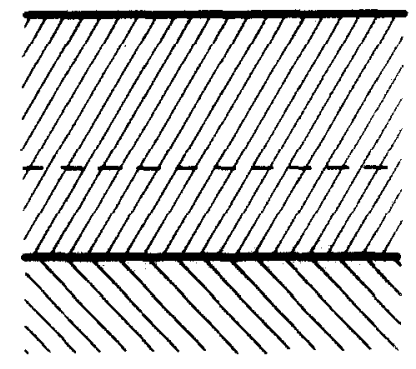


Another peculiarity observed is the second, slow growth mechanism. The initial (fast) silicon diffusion is therefore tentatively ascribed to silicon diffusion into the disordered regions in the evaporated titanium layer, whereas the final (slow) diffusion mechanism should correspond to the retarded diffusion of silicon in the ordered regions (grains).

\section{References}

I J. M. M. de Nijs and A. van Silfhout, Mater. Sci. Eng., B5 (2) (1990) 319 .

2 R. M. Tromp, G. W. Rubloff and E. J. van Loenen, I. Vac. Sici. Technol. A, 4(1986)865.

3 S. A. Chambers, D. M. Hill, F. Xu and J. H. Weaver, Phys. Rev. $B, 35(1987) 6.34$.

4 K. Holloway and R. Sinclair, I. Appl. Phys, 61 (1987) 1359.

5 I. J. M. M. Raaijmakers, Ph.D. Thesis, Eindhoven, 1988.

6 J. M. M. de Nijs, Ph.D. Thesis, University of Twente, Enschede, 1989.
7 J. M. M. de Nijs and A. van Silfhout, The Ti/c-Si solid state reaction III. The low temperature reaction kinetics, Appl. Surf. Sci, in the press.

8 K. Maex, Ph.D. Thesis, University of Leuven, 1988.

9 R. W. Balluffi and J. M. Blakely, Thin Solid Films, 25 (1975) 363

10 U. Gösele and K. N. Tu, J. Appl. Phys., 53 (1982) 3252.

11 J. M. Poate. K. N. Tu and J. W. Mayer, Thin FilmsInterdiffusion and Reactions. Wiley, New York. 1978.

12 M. del Giudice, J. J. Joyce, M. W. Ruckman and J. H. Weaver, Phys. Rev. B, 35(1987)6213.

13 K. N. Tu, G. Ottaviani, U. Gosele and H. Foll, J. Appl. Phys., $54(1983) 758$.

14 J. M. M. de Nijs, Thin Solid Films, $173(1989) 1$.

15 D. E. Aspnes, E. Kinsborn and D. D. Bacon, Phys. Rev. $B, 21(1980) 3290$

16 R. Fletcher, AERE-R 6799, 1971 (Theoretical Physics Division, AERE, Harwell).

17 L. S. Hung, J. Gyulai, J. W. Mayer, S. S. Lau and M.-A. Nicolet, J. Appl. Phys., $54(1983) 5076$.

18 M. Schneider. A. Rahman and I. K. Schuller, Phys. Rev. Lett., $55(1985) 604$.

19 K. H. Müller, Surf. Sci. Lett., 184 (1987) 375.

20 Y. Yamada and K. Yoshida, Appl. Surf. Sci., 33-34 (1988) 465.

21 K. N. Tu, J. Appl. Phys., 43(1972) 1303. 